\title{
The mechanism of pneumocephalus formation in patients with CSF fistulas
}

\author{
FRANCIS O WALKER,* BORIS A VERN†
}

From the Department of Neurology,* Wake Forest University, Bowman Gray School of Medicine, WinstonSalem, North Carolina, and Department of Neurology, $\dagger$ University of Illinois, Chicago, Illinois, USA

SUMMARY A novel but simple explanation of how pneumocephalus develops across CSF fistulas is presented and applied to an unusual case of pneumocephalus. Preexisting models of pneumocephalus formation are discussed and a substitute model is suggested.

Since the early descriptions of pneumocephalus numerous models have been proposed to explain how it develops across CSF fistulas. ${ }^{1-3}$ The inverted pop bottle model likens the formation of pneumocephalus to the process of air entering a fluid filled pop bottle that is suddenly inverted. The ball-valve model suggests that some CSF fistulas act as one-way valves, allowing air to enter the CSF compartment and trapping it there. Negative pressure models have suggested that in some cases, negative CSF pressure draws air into the CSF compartment. Although these models are helpful, pneumocephalus formation can be explained in a less complex fashion using known principles of CSF dynamics. A patient was recently studied who developed an unusually fulminant case of pneumocephalus from a lumbodermal CSF fistula. Using this case, an appropriate, simplified mechanism of pneumocephalus formation is presented with a related practical working model.

\section{Case report}

A 72-year-old woman was admitted for the abrupt onset of headache and confusion that occurred shortly after arising from bed. Ten years prior to admission she had undergone resection of a large recurrent lumbar paraspinal liposarcoma. Postoperatively she received 5000 rads of local radiation. An ulcer that later developed at the surgical site

Address for reprint requests: Francis O Walker, MD, Department of Neurology, Bowman Gray School of Medicine, Wake Forest University, 300 S Hawthorne Road, Winston-Salem, North Carolina 27103, USA.

Received 12 March 1985.

Accepted 12 June 1985

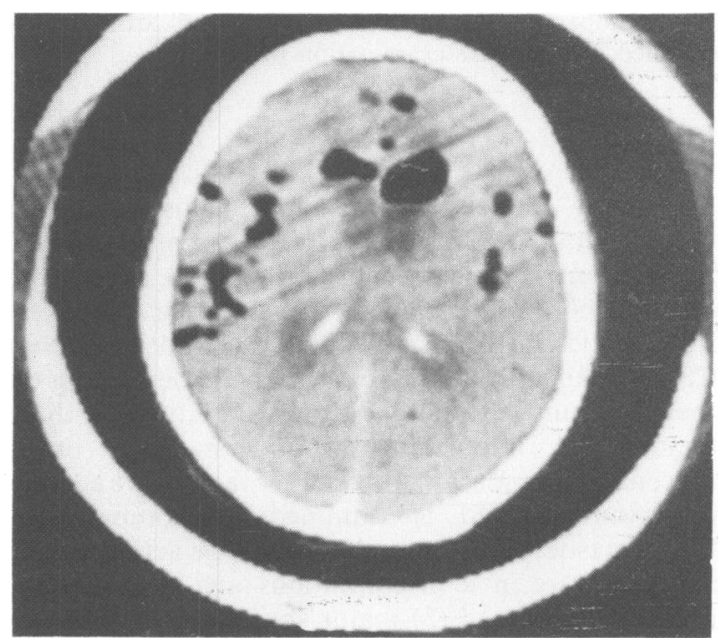

Fig 1 CT scan demonstrates air within the ventricles and subarachnoid spaces.

was refractory to conservative therapy. Surgical repair of the ulcer was declined and it grew slowly over the ensuing 7 years.

On the day of admission she was confused, combative, and had a stiff neck. A large $3 \mathrm{~cm}$ deep ulcer was present over her lumbar spine with 2 draining tracts at the base. CT scan revealed a large volume of ventricular and subarachnoid air (fig 1). Cisternal puncture yielded cloudy CSF from which Staphylococcus aureus was isolated. After her meningitis had responded to antibiotics, ytterbium was injected into the subarachnoid space via cisternal puncture, The rapid contamination of cotton pledgets placed in the ulcer cavity documented the suspected lumbodermal CSF fistula. After a second bout of meningitis she finally agreed to surgical 


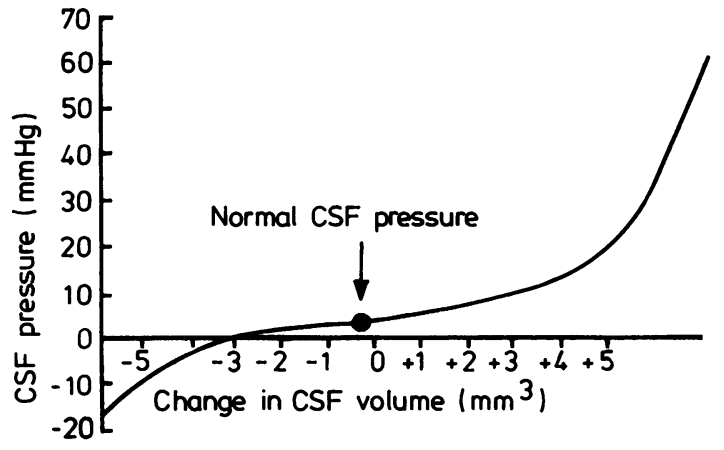

Fig 2 The CSF pressure-volume curve in dogs demonstrates the elastic properties of the CSF compartment. Changes in $C S F$ volume induce finite changes in CSF pressure. (Adapted from Lofgren ${ }^{4}$.)

repair of the fistula. During surgery a tear was found in the dura with 2 small protruding nerve roots; it communicated with the ulcer cavity. The defect was repaired with a dural graft and a skin flap was rotated over the wound. On the 12th postoperative day she had an unexpected cardiac arrest and died. Postmortem examination was refused.

\section{Discussion}

Preexisting models of pneumocephalus formation indicate that air tends to rise to the top of the cranial vault when it enters the subarachnoid space (the inverted pop bottle model), that negative CSF pressure can occur (the negative pressure model) and that CSF fistulas are subject to tamponade depending on their anatomy (the ball-valve model). Although these factors are important in understanding pneumocephalus formation, three other factors are equally important: (1) CSF is contained in a compartment that is elastic; (2) air and CSF exchange across a CSF fistula occurs in a discontinuous and not simultaneous fashion; and (3) major shifts in CSF pressure occur from CSF loss and changes in posture and intrathoracic pressure.

Figure 2 demonstrates the elastic properties of the CSF compartment. ${ }^{4}$ This CSF pressure-volume relationship is curvilinear in nature because the CSF compartment accommodates different volumes of CSF at different pressures. Note that in dogs CSF pressure falls below atmospheric pressure with $5 \mathrm{cc}$ of CSF loss. In hospital patients as little as $20 \mathrm{cc}$ of rapid CSF loss during lumbar puncture can generate negative (below atmospheric) CSF pressure. ${ }^{5}$

The calibre of most CSF fistulas is sufficiently small to prevent the simultaneous exchange of significant volumes of CSF and air; rather they exchange in a discontinuous fashion. Since the CSF compartment varies its volume and pressure, the movement of liq- uid and air across a fistula is determined by pressure $\frac{\bar{O}}{Z}$ gradients, as defined in Equation 1.67

$$
\mathrm{F}=\frac{\Delta \mathrm{P}}{\mathrm{R}} \text { where } \mathrm{R}=\frac{\mu}{\mathrm{a}^{2}} \mathrm{~K}
$$

where $F=$ Flow rate, $\Delta P=(C S F$ pressure at the fistula - air pressure at the fistula); $R=$ resistance, $\mathrm{a}=$ cross sectional area of the fistula, $\mu=$ viscosity $\frac{\rho}{\partial}$ and $\mathrm{K}$ is an empirically determined constant. (Although derived independently ${ }^{67}$ Equation $1 ₹$ paraphrases Ohm's law, $I=\frac{\Delta V}{R}$, in different units of $\underset{\overrightarrow{\vec{D}}}{\stackrel{\vec{\rho}}{\vec{g}}}$ measure.)

Equation 1 indicates that pneumocephalus can $\frac{\bar{O}}{\bar{O}}$ occur if and only if CSF pressure falls below atmos- $\frac{\bar{\rho}}{\partial}$ pheric pressure. Normally CSF pressure is greater $\stackrel{\mathbb{Q}}{\Omega}$ than air pressure so most fistulas lead to CSF extra- क vasation. If CSF loss exceeds net CSF production, the fistula will cause CSF volume and pressure to drop $\stackrel{\circ}{\circ}$ until CSF and atmospheric pressure equilibrate.

Once equilibrium develops across a CSF fistula any $\stackrel{\omega}{\sigma}$ further drop in CSF pressure will lead to pneumocephalus formation. In the lumbar region CSF ? pressure drops $300-400$ millimetres of $\mathrm{H}_{2} \mathrm{O}$ on $\overrightarrow{0}$ reclining from the upright position, ${ }^{8}$ and a similar i drop in pressure may have caused pneumocephalus î: i our patient. Conversely any transient increase in CSf pressure as provoked by straining or coughing cause a transient extravasation of CSF. On relaxation aa aliquot of air equal in volume to the lost CSF mus T enter the CSF compartment to reequilibrate pressure.

Davson's ${ }^{9}$ elastic model of the CSF compartme can be readily adapted to serve as a good practicis model of pneumocephalus formation. If a firm bü $\vec{\varphi}$ flexible plastic container is filled under pressure it will bulge. Should a small puncture be made near the bottom, water will exude until internal and external pressure equilibrate, without air entry. If it is then flipped on its side such that the puncture is at the middle or top of the container (analogous to a postural change), $\stackrel{\odot}{\varnothing}$ air will enter the container. Or, should the container be squeezed and released (analogous to a sneeze or cough) fluid will leak out and then will be replaced with air.

Although not incorporated in this model, internal tamponade is an important factor which may retard pneumocephalus formation. Fistulas at the cribiform plate or near the wall or floor of the middle cranial $\frac{5}{3}$ fossa are naturally tamponaded by the frontal and temporal lobes in the upright and head flexed posi- $\delta$ tions. Long, tortuous fistulas may close if exposed to $₹$ even slight suction. Aside from redundant nerve 을 roots, fistulas in the spinal subarachnoid space have no natural mechanisms of tamponade.

As can be seen from this analysis, any factors that predispose to CSF loss or to large fluctuations of CSF $\Omega$ 


\section{Table Factors predisposing to pneumocephalus}

1 Factors which promote CSF fistula formation:

2 Factors which lead to CSF loss and subsequent reduction in CSF pressure:

3 Factors which decrease CSF pressure by reducing CSF production or by decreasing intracranial intravascular volume:

4 Factors which increase or cause fluctuations in air pressure across CSF fistulas, particularly those in nasopharyngeal areas:

5 Factors which reduce the likelihood of tamponade

6 Factors which are rare causes of pneumocephalus in the absence of persistent CSF fistulas:
Trauma, surgery, infection or radiation in parameningeal areas

Large diameter or multiple CSF fistulas, Valsalva manoeuvre, or transient increase in CSF pressure from postural change

Diuretics, acetazolamide, dehydration, systemic hypotension, or Muller's experiment (the reverse of a Valsalva manoeuvre)

Sneezing, nose blowing, positive pressure resuscitators, or airplane flights

CFS fistulas of short length or that are distant from mobile CSF structures

Bacterial gas production in abcesses or diffusion of anaesthetic agents (Nitrous oxide)

pressure (or air pressure) across a fistula, or which minimise internal tamponade will facilitate pneumocephalus formation (table). In our patient, the location of the fistula exposed it to high CSF pressure when she was upright, causing rapid CSF loss. On reclining she exposed the fistula to a large drop in CSF pressure; in addition, the large calibre of her fistula and the absence of significant tamponade further predisposed to pneumocephalus formation. The combination of these factors readily accounts for her fulminant presentation; more importantly, the

absence of these predisposing factors in most cases of CSF fistulas accounts for the relative rarity of pneumocephalus.

\section{References}

${ }^{1}$ Markham JW. Pneumocephalus. In: Vinken PJ, Bruyn GW, eds. Handbook of Clinical Neurology. Vol 24, Injuries of the Brain and Skull. New York: Elsevier, 1976:201-13.

${ }^{2}$ Lunsford LD, Marroon JD, Sheptak PE, Albin MS. Subdural Tension Pneumocephalus. J Neurosurg 1979;50: 525-7.

${ }^{3}$ Markham JW. The clinical features of pneumocephalus based upon a survey of 284 cases with report of 11 additional cases. Acta Neurochir 1967;16:1-78.

${ }^{4}$ Lofgren J, von Essen C, Zwetnow NN. The pressurevolume curve of the cerebrospinal fluid space in dogs. Acta Neurol Scand 1973;49:557-74.

${ }^{5}$ Ryder HW, Espey FF, Kimbell FD, et al. The mechanism of the change in cerebrospinal fluid pressure following an induced change in the volume of the fluid space. $J$ Lab Clin Med 1953;41:428-35.

${ }^{6}$ Portnoy HD, Tripp L, Croissant PD. Hydrodynamics of shunt valves. Childs Brain 1976;2:242-56.

${ }^{7}$ Hakim S, Durancela-Roche F, Burton JD. A critical analysis of valve shunts used in the treatment of hydrocephalus. Dev Med Child Neurol 1973;15:232-55.

${ }^{8}$ Loman J. Components of cerebrospinal fluid pressure as affected by changes in posture. Arch Neurol 1934; 31:674-81.

${ }^{9}$ Davson H. Physiology of the Cerebrospinal Fluid. Boston: Little Brown and Company, 1967:337-432. 